\title{
ALGEBRAS WHICH DO NOT POSSESS A FINITE BASIS*
}

BY

\section{J. H. M. WEDDERBURN}

1. Introduction. The object of this paper is to classify algebras which do not have a finite basis. The methods used are similar to those employed in a former paper, $t$ but considerable difficulty was experienced in extending the results of this paper, as the proofs of many of the principal theorems depended on the use of induction and were therefore tied up with the finiteness of the basis; and, in fact, these difficulties have been only partially overcome, as is shown by the postulates assumed in $\S 5$. It is hoped, however, that, in spite of their incompleteness, the results presented here will be found of sufficient interest to justify their publication.

It is noteworthy how little place the finiteness of the basis-or indeed the presence of any basis at all-has in the principal theorems of linear algebras. The first theorem of importance in which it seems to be required is that in which it is shown that primitive idempotent elements exist in an algebra which possesses elements of finite rank that are not nilpotent; and in two other cases it has not been found possible to complete the argument when a finite basis is not assumed, namely, in the theorems which state that, if an algebra is not nilpotent, it contains an idempotent element, and that the maximal nilpotent invariant subalgebra can be separated from the rest of the algebra.

The proofs of many of the theorems parallel those for the case of algebras with a finite basis very closely, so closely in fact that it might have been sufficient to refer the reader to previous treatments of the subject. It has been thought advisable, however, to repeat most of these proofs, as otherwise the reader would feel much uncertainty as to the logical completeness of the treatment. In one or two cases reference has been made to the paper mentioned above, or to Professor L. E. Dickson's treatise $₫$ in place of giving a detailed proof.

It was found inconvenient to give in one section all the postulates used as in several cases their statement involved some previous discussion. After a short discussion in $\S 2$ of algebras defined in the manner used by Hamilton,

- Presented to the Society, May 3, 1924.

†Proceedings of the London Mathematical Society, ser. 2, vol. 6 (1907), pp. 77-118; this paper is cited hereafter as W.

$\Varangle$ L. E. Dickson, Algebras and their Arithmetics, Chicago, 1923, cited hereafter as D. 
the postulates common to all associative algebras are given in $\S 3$ while those peculiar to algebras which do not have a finite basis are given in $\S 5$.

2. The Hamiltonian definition. Hamilton's definition* of a linear associative algebra may be modified as follows. Let $t$ be a variable which runs through a given range or set of values $G$ (which need not be numerical although this will generally be the case) and $\xi(t)$ a single-valued function which is defined for every value of $t$ in $G$ and which has values, for the present restricted to be finite, which lie in a given fieldt $F$. Two such functions, $\xi(t)$ and $\eta(t)$, are said to be equal if, and only if, $\xi(t)=\eta(t)$ for every value of $t$ in $G$. The sum $\xi+\eta$ is the ordinary sum in the field $F$; the product $\xi(t) \times \eta(t)$ may not be the ordinary product but is to be defined in any particular case subject to the following conditions:

$$
\begin{gathered}
\xi \times(\eta \times \zeta) \cdots(\xi \times \eta) \times \zeta, \\
\xi \times(\eta+\zeta)=\xi \times \eta+\xi \times \zeta, \quad(\eta+\zeta) \times \xi=\eta \times \xi+\zeta \times \xi .
\end{gathered}
$$

We shall also assume that the product of two functions in a given set belongs to the same set. If this condition does not hold in a given set, as in Grassmann's calculus, the set may always be so extended that it has this property.

If $x(t, \alpha)$ is a function of $t$ and $\alpha$ in $G$ such that

$$
x(t, \alpha)= \begin{cases}0, & \alpha \neq t, \\ 1, & \alpha=t,\end{cases}
$$

then

$$
\xi(\alpha) x(t, \alpha)= \begin{cases}0, & \alpha \neq t, \\ \xi(t), & \alpha=t .\end{cases}
$$

We may therefore set

$$
\xi(t)=\sum_{\tau(\boldsymbol{\alpha})} \xi(\boldsymbol{\alpha}) x(t, \alpha) .
$$

Here the exact meaning of the $\Sigma$ is best left somewhat indefinite, a special definition being given in any particular case; the properties required of it are detailed in the next section and in the meantime it suffices to give two

\footnotetext{
* See the introduction to his Lectures on Quaternions, Dublin, 1853; also Transactions of the Royal Irish Academy, vol. 17 (1835), pp. 293-422, vol. 21 (1843), pp. 199-296.

$\dagger$ This may be generalized considerably by taking in place of $F$ some linear associative algebra already defined (so giving the direct product) or even an algebra such as the algebra of logic.
} 
examples. If the elements of the range $G$ form an enumerable set, $\sum_{G}$ denotes ordinary summation, of which algebras with a finite basis form a particular case, other examples being given in $\S 9$. Again, if $G$ is an interval of the real continuum, $\sum_{G(\alpha)} \xi(\alpha) x(t, \alpha)$ may be defined as the Stieltjes integral $\int_{G} d(\xi(\alpha) x(t, \alpha))$ if $\xi \xi(\alpha)$ is properly restricted.

Any set of functions $e_{t}(\alpha), \alpha$ in $G$, are said to form a linearly independent set in $G$ if every relation of the form

$$
\sum_{G(\alpha)} \xi(\boldsymbol{\alpha}) e_{t}(\boldsymbol{\alpha})=0
$$

for every $t$ in $G$ entails $\xi(\alpha) \equiv 0$ in $G$. If, further, every function of the algebra can be expressed in the form

$$
\xi(t)=\sum_{G(\alpha)} f(\alpha) e_{t}(\alpha)
$$

the set $e_{t}(\alpha)$ is said to form a basis of the algebra, and the cardinal number of $G$ is called the order of the algebra.

When a basis is used to define an algebra, the functional notation is not usually convenient. On the analogy of algebras with a finite basis we shall generally write (2.4) in the form

$$
x=\sum_{G(\alpha)} \xi(\alpha) x_{\alpha} .
$$

The values of $\xi(\alpha)$ are called the coefficients of $x$ and, if $\lambda$ is any constant mark of the field $F$, we shall set

$$
\lambda x=\sum_{G(\alpha)} \lambda \leqq(\alpha) x_{\alpha} .
$$

When this point of view is adopted, the product of $x_{r}$ and $x_{s}$ will be denoted by $x_{r} x_{s}$.

The condition that the product is associative may be stated in much the same way as when a finite basis exists. Since we are assuming that $x_{r} x_{s}$ lies in the given set, we must have

$$
x_{r} x_{s}==\sum_{G(t)} k(r, s, t) x_{t},
$$

where $l:(r, s, t)$ is some function defined for $r, s, t$ in $G$, and, if multiplication is associative, we find in the usual manner

$$
\sum_{G(\alpha)} k_{r s \alpha} k_{\alpha \beta \beta}=\sum_{G(\alpha)} k_{r \alpha t} k_{s \beta \alpha} .
$$


If the units used are the Hamiltonian ones described above, equation (2.5) may be written

$$
x_{r}(\boldsymbol{\alpha}) \times x_{s}(\boldsymbol{\alpha})=k(r, s, \boldsymbol{\alpha}),
$$

or, for any functions $\xi$ and $\eta$ of the set

$$
\xi(\boldsymbol{\alpha}) \times \eta(\boldsymbol{\alpha})=\sum_{r, s} \xi(r) \eta(s) x_{r}(\boldsymbol{\alpha}) \times x_{s}(\boldsymbol{\alpha})=\sum_{r, s} \xi(r) \eta(s) k(r, s, \boldsymbol{\alpha}) .
$$

It is then clear that we may take as a particular interpretation of $\Sigma$ (or as our definition of the product of two functions)

$$
\xi(\boldsymbol{\alpha}) \times \eta(\boldsymbol{\alpha})=\iint \xi(r) \eta(s) k(r, \varepsilon, \alpha) d r d s
$$

with

$$
\int k(r, s, t) k(t, \boldsymbol{\beta}, \boldsymbol{\alpha}) d t=\int k(r, t, \boldsymbol{\alpha}) k(s, \boldsymbol{\beta}, t) d t
$$

as the eondition of associativity.*

Examples of functions $k(r, s, t)$ which satisfy (2.73) are easily constructed by employing orthogonal functions in conjunction with the constants of an algebra with an enumerable basis. The following illustrations are constructed from the constants of ordinary complex numbers and quaternions:

$$
\begin{aligned}
k(r, s, t)=\sum_{m} k_{m} & \sin m(r+s-t) \\
k(r, s, t)=\sum_{m} k_{m} & \{[\sin (2 m+1) r \sin (2 m+1) s \\
& -\sin (2 m+2) r \sin (2 m+2) s] \sin (2 m+1) t \\
& +[\sin (2 m+1) r \sin (2 m+2) s \\
& +\sin (2 m+2) r \sin (2 m+1) s] \sin (2 m+2) t\}
\end{aligned}
$$

$$
\begin{aligned}
k(r, s, t)=\sum_{m} k_{m} & \{\sin (2 m+1)(r+s-t) \\
& +\sin [(2 m+1) r+(2 m+2)(s-t)] \\
& -\sin [(2 m+2) r-(2 m+1) s-(2 m+2) t] \\
& +\sin [(2 m+2) r-(2 m+2) s-(2 m+1) t]\} .
\end{aligned}
$$

* It will often be necessary here to replace ordinary integration by one of its many generalizatious and suitable restrictions must, "of course, be placed on the functions involved. 
Here the constants $k_{m}$ are only restricted by considerations of convergence and integrability, and the range of each of the variables is over a period $2 \pi$. It is clear that, subject to these conditions, (2.81) represents any odd periodic function of $r+s-t$ with period $2 \pi$, so that the operation

$$
\xi(\alpha) \times \eta(\alpha)=\int_{-1}^{1} \int_{-1}^{1} \xi(r) \eta(s) f(r+s-\alpha) d r d s
$$

defines an algebra in the range -1 to +1 when $f(t)$ is an odd periodic function with the period 2 .

3. Fundamental postulates and definitions. We shall now give a more abstract definition by means of postulates without attempting, however, to make these independent, the aim being descriptive rather than analytical.

A linear associative algebra $A$ is a set of two or more elements $a, b, c, \cdots$ subject to two operations, namely, addition, which will denoted by + , and multiplication, which will be denoted simply by juxtaposition of the factors. These operations are subject to the following conditions.

Postulate 3.11 .

$$
\begin{aligned}
& A_{1}: a+b \text { is an element of } A ; \\
& A_{2}: a+b=b+a ; \\
& A_{3}: a+(b+c)=(a+b)+c ; \\
& A_{4}: \text { There is an element } 0 \text { such that } a+0=a \text { for every element } a \text { of } A ; \\
& A_{5}: \text { For every element } a \text { there exists an element } b \text { such that } a+b=0 ; \\
& M_{1}: a b \text { is an element of } A ; \\
& M_{2}: a \cdot b c=a b \cdot c ; \\
& A M: a(b+c)=a b+a c, \quad(b+c) a=b a+c a .
\end{aligned}
$$

It is easily seen that 0 is unique in $A_{4}$ and that $b$ in $A_{5}$ is uniquely determined when $a$ is given; $b$ is denoted by $-a$ and $-(-a)=a$.

If $a$ is any element, $a+a$ is denoted by $2 a$ and in general $a+a+\cdots+a$ ( $m$ terms) is written $m a$; evidently $(m a) b=a(m b)=m(a b)$. When $a \neq 0$, it is not difficult to show that the smallest integer for which $m a=0$, if such an integer exists, is always a prime; we shall assume that this prime, if it exists, is the same for all elements since, when this is not the case, the algebra is reducible. When no such integer exists, we assume the following postulates.

Postulate 3.12. If $a$ is an element of $A$ different from 0 and $m$ is a positive integer, there exists an element $b$ such that $a=m b$.

The element $b$, which is unique, will be denoted by $(1 / m) a$. This postulate is sufficient for many purposes, but the following one, which includes it, will usually be more convenient. 
Posrulate 3.13. (i) There is associated with A a field $F$ such that to any non-zero element a of $A$ there is allied a subset $A_{a}$ of $A$ which is in $(1,1)$ correspondence with the elements of $F, a$ and 0 in $A_{a}$ corresponding respectively to 1 and 0 in $F$; the element corresponding to a mark $\xi$ of $F$ is denoted by $\xi a$. The correspondence is preserved under the operation of addition, that is, $\boldsymbol{\xi}_{1} a+\xi_{2} a=\left(\xi_{1}+\xi_{2}\right) a$;

(ii) If $b \neq 0$ is any element of $A_{a}$, then $A_{b}=A_{a}$ and, if $\xi_{2} b=\xi_{1} a\left(\xi_{2} \neq 0\right)$, then $a=\left(\xi_{1} \xi_{2}^{-1}\right) b$;

$$
\begin{gathered}
\xi_{3}\left(\xi_{1} a+\xi_{2} b\right)=\xi_{3} \xi_{1} a+\xi_{3} \xi_{2} b ; \\
\xi a \cdot b=a \cdot \xi b=\xi(a b) .
\end{gathered}
$$

This composite postulate is broadly equivalent to saying that the elements of $A$ correspond to an affine geometry in which these elements are the points of the geometry, or to a projective geometry in which the sets $A_{a}$ correspond to points.

The combined postulates 3.11, 12 and 13 will be referred to as Postulate 3.1. In these postulates we have considered combinations of elements by a finite number only of applications of the fundamental operations. Later we shall see that infinite sums are required in certain cases which are introduced by postulates as required. We have, however, to frame our definitions from the start so as to admit the possibility of such combinations of an infinite number of terms and it is therefore necessary to detail the properties required of the summation sign $\Sigma$. For the present we shall merely say that, if $x_{t}$ is a set of elements of $A$ in $(1,1)$-correspondence with a range or set of values of a variable $t$, then for certain ranges-which depend on the particular algebra under discussion-there exist elements denoted by $\sum_{G} \xi_{t} x_{t}$, where $\xi_{t}$ is a function of $t$ in $G$ whose values lie in $F$, and the summation sign $\sum$ has the following properties:

(a) If $G$ contains only a finite number of elements $1,2, \cdots, n, \sum_{G} \xi_{t} x_{t}$ denotes $\xi_{1} x_{1}+\xi_{2} x_{2}+\cdots+\xi_{n} x_{n}$

(b) Whether $G$ is finite or not, if $y_{t}$ is also a set of elements of $A$ defined for $t$ in $G$ and $z$ is any element of $A$, then

$$
\begin{gathered}
\sum_{G}\left(\xi_{t} x_{t}+\eta_{t} y_{t}\right)==\sum_{G} \xi_{t} x_{t}+\sum_{G} \eta_{t} y_{t} \\
z \sum_{G} \xi_{t} x_{t}=\sum_{G} \xi_{t} z x_{t}, \quad \sum_{G} \xi_{t} x_{t} \cdot z==\sum_{G} \xi_{t} x_{t} z
\end{gathered}
$$

provided always that in each case the summations used have a meaning. 
When $\sum_{G} \xi_{t} x_{t}$ exists in $A$, we shall say that it is linearly dependent on $x_{t}, t$ in $G$.

A complex* in $A$ is any subset of $A$ which is closed under the operation of addition but not necessarily under that of multiplication. If $B$ and $C$ are complexes such that every element of $B$ is an element of $C$ and vice versa, we write $B=C^{r}$; if $B$ contains all the elements of $C$ and also elements not in $C$, we write $B>C$ or $C<B$. If the order of a complex is 1 , that is, if when $b$ is any non-zero element of $B$ all elements of it have the form $\xi b, \xi$ an element of $F$, we shall write $B=(b)$ or, when there is no risk of confusion, $B=b$; thus $x<B$ means that $x$ is an element of $B$. The intersection of two complexes $B$ and $C$ is the complex of all elements common to both; it is denoted by $B-C^{r}$.

If $B$ and $C$ are two complexes, the complex of all elements which can be derived from the elements of $B$ and $C^{r}$ by means of the operation of addition is called the sum of $B$ and $C$ and is written $B+C$. Evidently the addition so defined is commutative and associative. Similarly, if $B_{t}, t$ in a range $G$, is a set of complexes, $\sum_{G} B_{t}$ is the complex of all elements derivable from the totality of elements in the $B_{t}$ by means of the operation of addition. Even if $G$ is an infinite range, this does not necessarily involve infinite sums of elements.

If $x$ and $y$ are variable elements of $B$ and $C$ respectively, the totality of elements of the form $x y$ together with those elements derivable from them by the operation of addition is called the product of $B$ into $C$ and is written $B C$. The multiplication so defined is associative and distributive. We may also note here that

$$
\begin{gathered}
A-\left(B-C^{\prime}\right)=(A-B)-C, \\
A\left(B-C^{\prime}\right) \leqq A B-A C, \quad(B-C) A \leqq B A-C A .
\end{gathered}
$$

If $C$ is a subcomplex of a complex $B$, any two elements $x_{1}, x_{2}$ of $B$ for which $\left(x_{1}-x_{2}\right)<C$ are said to be congruent modulo $C$ and we write $x_{1} \equiv x_{2}(\bmod C) ;$ all elements congruent to $x_{1}$ modulo $C$, that is, all elements of the form $x_{1}+y$ where $y<C$, are said to form a classt modulo $C$. The class corresponding to $x_{1}$ may be written $\left[x_{1}\right]$; it is completely determined when any one of its elements is given. The class [0] is the complex $C^{r}$ itself.

\footnotetext{
* This term was introduced into the theory of tinite groups by Frobenius in a similar sense. Dickson uses the term "linear set", Scorza, "linear system".

$\dagger$ Cf. D, p. 80 .
} 
Two complexes $D$ and $E$ are said to be congruent modulo $C$ if there is a $(1,1)$-correspondence betwecia their elements such that, if $x$ and $y$ are corresponding elements of $C$ and $D$ respectively, then $x-y<C$.

If a complex $B$ has a finite basis, it is clear that, when $C$ is a proper subcomplex of $B$, there exists a complex $D$ such that $B=C+D, C \frown D=0$; but here, as we do not assume the existence of any basis, it is necessary to have the following postulate:

Postulate 3.2. If $C$ is a subcomplex of a complex $B$, there exists a complex $D$ which has no element in common with $C$ and for which $B=C+D$.

The complex $D$ is called a supplement of $C$ in $B$.

A few definitions are conveniently given here. Any element different from 0 which is equal to its own square is said to be idempotent. If $e$ is idempotent and $x$ an element of $A$, then, if there exists an element $y$ such that $x y=e(y x=e), x$ is said to have a right (left) inverse with respect to $e$; if $x$ has neither a right nor a left inverse with respect to $e$, it is said to be singular with respect to $e$. It may be noticed here that, if $x y=e$ is idempotent, then yex is also idempotent. If there is an element $m$ such that $m x=x=x m$ for every element of $A$, it is called the modulus of $A$; it is evidently unique. If some power of an element is 0 , it is said to be nilpotent, and, if $x^{n}$ is the lowest power of $x$ which is $0, n$ is called the index of $x$.

If there exist in a complex $B$ a set of elements $x_{t}, t$ in a range $G$, such that (i) every element of $B$ has the form $\sum_{G} \xi_{t} x_{t}$, the $\xi$ 's being elements of the field, and (ii) $\sum_{G} \xi_{t} x_{t}=0$ if, and only if, $\xi_{t}=0$ for every $t$ in $G$, then the set $x_{t}$ is called a basis of $B$. The cardinal number of the set $G$ is called the order of the basis and, if this number is unique, it is called the order of the complex. The existence of a basis is not assumed in this paper, but all the examples constructed so far possess one.

The algebra generated by the elements $x_{1}, x_{2}, x_{3}, \ldots$ is denoted by $\left\{x_{1}, x_{2}, \cdots\right\}$; the order of $\{x\}$ is called the rank of $x$.

Integral powers of a complex are defined in the usual manner; thus $B \cdot B^{m-1}=B^{m}=B^{m-1} \cdot B$. The condition that a complex is an algebra then takes the form $B^{2} \leqq B$. A phenomenon occurs here in the case of algebras which do not have a finite basis which is not present when the order is finite. Let $A$ be the algebra generated by $a, b, c$ where

$$
a b=c, \quad b a=0, \quad a c=c b=c^{2}=0 .
$$

If $A_{1}=\{a\}, A_{2}=\{b\}$, then

$A=A_{1}+A_{2}+(c), \quad A_{1} A_{2}=(c), \quad A_{2} A_{1}=A_{1} c=c A_{2}=c A_{1}=A_{2} c=0$, 
and hence

$$
A^{n}=A_{1}^{n}+A_{2}^{n}+(c) \text {. }
$$

The complex $C=(c)$ is therefore common to all integral powers of $A$ and it is clearly the only such complex; we therefore write

$$
A^{\omega}=\lim _{n=\infty} A^{n}=C
$$

Since $C^{2}=0$, we have $A^{\omega \cdot 2}=0$. Similarly in the algebra defined by

$$
\begin{gathered}
a b=c, \quad b a=d, \quad a c=c=c b, \quad d a=d=b d, \\
A_{1}=\{a\}, \quad A_{2}=\{b\}, \quad B=\{c, d\},
\end{gathered}
$$

we have $A^{\omega}=B$ and $A^{\omega^{2}}=B^{\omega}=0$.

The smallest ordinal number $\nu$ for which $\left(A^{\nu}\right)^{2}=A^{\nu}$ is called the index* of $A$. For instance, in the second example given above the index of $A$ is $\omega^{2}$ and that of $B$ is $\omega$. If $A^{\nu}=0$, as in this example, $A$ is said to be nilpotent.

4. Invariant subalgebras. $A$ complex $B$ in an algebra $A$ such that $A B \leqq B, B A \leqq B$ is itself an algebra, and it is said to be an invariant subalgebra of $A$. The first two theorems regarding such subalgebras are proved in exactly the same way as when there is a finite basis and hence they are merely stated here.

ThEOREM 4.1. If $B$ is a proper invariant subalgebra of an algebra $A$, an algebra can be derived from $A$ by regarding as equivalent those elements of $A$ which differ only by an element of $B+$.

This algebra is called the difference algebra of $A$ and $B$ and is denoted by $A-B$. To any algebraic identity in $A-B$ there corresponds a congruence in $A$ modulo $B$.

THEOREM 4.2. If $B_{1}$ and $B_{2}$ are proper invariant subalgebras of $A$, and $B_{1}>B_{2}$, then $A-B_{2}$ has an invariant subalgebra which is simply isomorphic with $B_{1}-B_{2}$, and conversely $\ddagger$

* When $\nu$ is finite, it is easily shown that this definition is equivalent to saying that $\nu$ is the smallest integer for which $A^{\nu+1}=A^{\nu}$.

$\dagger$ Cf. W, p. 82; D, p. 39 .

$\ddagger$ Cf. D, p. 41 . 
An algebra which has no proper invariant subalgebra is said to be simple. If $B$ is a maximal invariant subalgebra of $A, A-B$ is simple and conversely.

An algebra is said to be the direct sum of two proper subalgebras $A_{1}, A_{2}$ if

$$
A=A_{1}+A_{2}, \quad A_{1} A_{2}=0 \quad 0 \quad A_{2} A_{1}, \quad A_{1}-A_{2}=0
$$

and, when such a form for $A$ exists, it is said to be reducible. When (4.1) holds, we shall write $A=A_{1} \oplus A_{2}$ in place of $A_{1}+A_{2}$ when it is desired to indicate that $A$ is reducible; the component parts of the sum will be referred to as recluced parts of $A$. A reduced part is evidently an invariant subalgebra.

Theorem 4.3. If an algebra $A$ has a proper invariant subalgebra $B$ that possesses a modulus, $A$ is reducible.

Let $C^{\prime}$ be a supplement of $B$ in $A$ so that

$$
A=B+C^{\prime}, \quad B-C^{\prime}=0
$$

also let $e_{1}$ be the modulus of $B$ and $y^{\prime}$ a variable element of $A$. If we set

$$
y=y^{\prime}-e_{1} y^{\prime}-y^{\prime} e_{1}+e_{1} y^{\prime} e_{1}
$$

then, as $y^{\prime}$ varies in $A, y$ evidently traces out a complex $C^{\prime}$ in $A$. This complex is congruent to $C^{\prime}$ modulo $B$ since, $B$ being invariant, $e_{1} y^{\prime}, y^{\prime} e_{1}, e_{1} y^{\prime} e_{1}$ are elements of $B$, and $y=0$ if, and only if, $y^{\prime}<B$; hence $A=B+C$, $B-C=0$.

If $x$ is any element of $B$,

$$
x y=x y^{\prime}-x e_{1} y^{\prime}-x y^{\prime} e_{1}+x e_{1} y^{\prime} e_{1}=0
$$

since $x e_{1}=x$; therefore $B C \cdots 0$, and similarly $C B=0$. Since $B$ has a modulus, this shows that $C$ is an algebra, which also follows from $e_{1} C=0=C e_{1}$, so that, if $y$ and $y_{1}$ are any elements of $C$,

$$
y y_{1}=y y_{1}-e_{1} y y_{1}-y y_{1} e_{1}+e_{1} y y_{1} e_{1}<C
$$

by (4.2). The theorem then follows from the definition of reducibility.

Corollary. The algebra $C$ is unique.

For, if $A=B \oplus D$, every element $x$ of $D$ has the form $y+z$, where $y<B, z<D$ and $0=e_{1} x=e_{1} y+e_{1} z=y$ since $e_{1} B=B$. Hence every element of $D$ belongs to $C$ and conversely. 
As a converse to the preceding theorem we have the following

Theorem 4.4. If $A=B \oplus C$ has a modulus, so have also $B$ and $C$.

For, if $e$ is the modulus of $A$, we have $e=e_{1}+e_{2}$ where $e_{1}<B, e_{2}<C^{\prime}$, and if $x<B$, then

$$
e_{1} x=\left(e-e_{2}\right) x=x-e_{2} x-x,
$$

since $e_{\mathbf{2}} x<B-C^{r}=0$.

The following two minor theorems are occasionally useful.

Theorem 4.5. If $A_{1}$ and $A_{2}$ are algebras such that $A_{1} A_{2}=0=A_{2} A_{1}$, and if either $A_{1}$ or $A_{2}$ has a modulus, then $A_{1}-A_{2}=0$.

For, if $A_{1}$ has a modulus $e_{1}$ and $B=A_{1} \frown A_{2}$, then $e_{1} B=B$ since $B<A_{1}$, and $e_{1} B=0$ since $B<A_{2}$; hence $B \cdots 0$.

Theorem 4.6. If $A=A_{1} \oplus B_{1}=A_{2} \oplus B_{2}$, and if $A_{1}$ and $A_{2}$ are irreducible and each has a modulus, then either $A_{1}=A_{2}$, or $A_{1} A_{2}=0=A_{2} A_{1}$ $=A_{1} \frown A_{2}$.

Let $e_{1}$ be the modulus of $A_{1}$ and $e_{2}$ that of $A_{2}$. Since $A_{1}$ and $A_{2}$ are invariant and each has a modulus, it follows that

$$
A_{1} A_{2} \quad A_{1}-A_{2} \quad A_{2} A_{1} \text {. }
$$

Hence $e_{1} e_{2}<A_{1}-A_{2}$ and is consequently its modulus. But by Theorem 4.3, $A_{1}$, being irreducible, cannot have a proper invariant subalgebra with a modulus; hence either $A_{1}==A_{2}$ or $A_{1}-A_{2}=0$.

5. Idempotent elements. The theory of idempotent elements is somewhat more elusive in the case of infinite algebras than in that of algebras with a finite basis; and certain difficulties arise which it has not proved possible, so far, to overcome except by restricting the class of algebra considered by further postulates.

If $e$ is an idempotent element of an algebra $A$, it is the modulus of $e A e$; and, if $e A e$ contains no idempotent element besides $e$, the latter is said to be primitive. When $e$ is not primitive, $e A e$ then contains at least one idempotent element $e^{\prime}$, which is necessarily commutative with $e$ so that $e^{\prime \prime}==e-e^{\prime}$ is also idempotent and $e^{\prime} e^{\prime \prime}=0=e^{\prime \prime} e^{\prime}$. More generally, if $e_{s}$ and $e_{t}$ are commutative idempotent elements, then $e_{s} e_{t}, e_{s}-e_{s} e_{t}, e_{t}-e_{s} e_{t}$ are all idempotent (unless one of them is 0 ), the product of any two of them is 0 , and the complex formed from them contains both $e_{s}$ and $e_{t}$. Further, since $e_{s} e_{t}$ is contained in both $e_{s} A e_{s}$ and $e_{t} A e_{t}$, it follows that $e_{s} e_{t}=0$ when $e_{s}$ and $e_{t}$ are primitive; hence in a set of primitive idempotent elements which are commutative with each other the product of any two is necessarily zero. 
When an algebra $A$ has a finite basis, it is readily proved that primitive idempotent elements exist whenever there is some element in $A$ which is not nilpotent. Our postulates for infinite algebras, however, are not sufficiently strong to enable us to draw similar conclusions as is seen from example 9.7, in which it can be shown that no idempotent element exists except when certain infinite series of elements are admitted as elements of $A$. We therefore assume the following postulate.

Postulate 5.1. An algelra which contains an idempotent element possesses at least one primitive idempotent element.

Let us suppose that $A$ contains a primitive idempotent element $e_{1}$, and let $e_{t}, t$ in a range $G$, be the set of all primitive idempotent elements which are commutative with $e_{1}$ and with each other; it then follows as above that $e_{r} e_{s}=0$ when $r \neq s$. Such a set is called a complete primitive complementary set, and $e=\sum_{G} e_{t}$, if it exists, is called a principal idempotent of $A$. Any set of idempotent elements $e_{t}$, primitive or not, for which $e_{r} e_{s}=0(r \neq s)$, will be called a complementary set.

If $e$ is any idempotent element and $x$ a variable element of $A$, then

where

$$
x=x_{0}+x_{1}+x_{2}+x_{3}
$$

$x_{0}=x-e x-x e+e x e, \quad x_{1}=e x-e x e, \quad x_{2}=x e-e x e, \quad x_{3}=e x e$.

As $x$ runs through the elements of $A$, the elements $x_{0}$ evidently form a complex which we shall denote by $A_{00}=\sum_{x} x_{0}$, and we have similarly the complexes

$$
A_{10}=\sum_{x} x_{1}, \quad A_{01}=\sum_{x} x_{2}, \quad A_{11}=\sum_{x} x_{3}=e A e .
$$

These complexes are obviously supplementary and

$$
A=A_{00}+A_{10}+A_{01}+A_{11} \text {. }
$$

This is called the Peirce decomposition of $A$ relatively to $e ; A_{00}+A_{10}$ is the complex of all elements $y$ of $A$ for which $y e=0, A_{00}+A_{01}$ is the complex of elements for which $e y=0$, and $A_{00}$ the complex for which $e y=0=y e .^{*}$

Before extending this decomposition to the case where a complete complementary set replaces $e$, we require the following postulates.

\footnotetext{
${ }^{*}$ It is sometimes convenient to note that, if $B=\sum_{x}(e x-x e)$, then $A_{01}=B e$ and $A_{10}=e B$.
} 
Postulate 5.2. If $e_{t}, t$ in $G$, is a complementary set of idempotent elements, the element $e=\sum_{G} e_{t}$ exists in $A$ and, if $x$ is any element of $A$, then

$$
e x=\sum_{G} e_{t} x, \quad x e=\sum_{G} x e_{t}, \quad e x e=\sum_{G} \sum_{G} e_{s} x e_{t} .
$$

Postulate 5.3. If $x_{t}, t$ in $G$, is a set of elements of $A$ such that $x=\sum_{G} x_{t}$ exists, then

$$
y x=\sum_{G} y x_{t}, \quad x y=\sum_{G} x_{t} y
$$

for every element $y$ of $A$.

Let $e_{t}, t$ in $G$, be a complementary set of idempotent elements, and put $e=\sum_{G} e_{t}$ in (5.1). In view of the postulates just given we may set

or, if

$$
x_{1}=\sum_{G}\left(e_{t} x-e_{t} x e\right), \quad x_{2}=\sum_{G}\left(x e_{t}-e x e_{t}\right), \quad x_{3}=\sum_{G} \sum_{G} e_{s} x e_{t}
$$

$$
A_{t 0}=\sum_{x}\left(e_{t} x-e_{t} x e\right), \quad A_{0 t}=\sum_{r}\left(x e_{t}-e x e_{t}\right), \quad A_{s t}=e_{s} A e_{t},
$$

then

$$
A=A_{00}+\sum_{G} A_{t 0}+\sum_{G} A_{0 t}+\sum_{G} \sum_{G} A_{s t}
$$

where the intersection of any two complexes is zero.

If the set $e_{t}$ is a complete primitive complementary set, $e$ is a principal idempotent element of $A$, and $A_{00}$ then contains no idempotent element, as otherwise $e_{t}$ would not be a complete set. If $A$ has a modulus, it equals $e$ and $A_{00}, A_{10}$ and $A_{01}$ are 0 .

Before proceeding further we must consider more closely the nature of individual elements of $A$. When a finite basis exists, either for $A$ itself or for some subalgebra which is not nilpotent, then $A$ contains an idempotent element; but, when no such basis exists, the usual proofs break down. A closely related theorem is that, if $e$ is the only idempotent element in $A$, every element which has no inverse with respect to $e$ is nilpotent, and that the totality of such elements forms a nilpotent invariant subalgebra. The proof of this theorem, in one method of attack at least, leads to an equation of the form

$$
e=x+y
$$

where neither $x$ nor $y$ has an inverse with respect to the primitive idempotent element $e$. If $x$ is a nilpotent element of index $n$ for wlich $e x=x=x e$, then

$$
y\left(e+x+x^{2}+\cdots\right) \equiv(e-x)\left(e+x+x^{2}+\cdots\right)=e ;
$$


which is impossible since we have assumed that $x$ has no inverse. If $x$ is not nilpotent, or if $n$ is not finite, this method of proof requires the existence of the infinite series

$$
e+x+x^{2}+\cdots
$$

as an element of $A$. But, if we assume that this element does exist, certain difficulties arise. If $z=e-x$, we should naturally expect that the algebras generated by $x$ and $z$ respectively would be simply isomorphic since the elements of their bases have the same law of combination; but in spite of this isomorphism we cannot assume the existence of

$$
w=e+z+z^{2}+\cdots
$$

since then $w x=w(e-z)=e$, whereas $x$ has no inverse; and, moreover, the element $v$, although an element of $\{e, x\}$, cannot be expressed in terms of the basis $e, x, x^{2}, \cdots$, at least with finite coefficients. The nature of an element $x$, therefore, cannot be predicted from the laws of combination of $\{x\}$ alone, but the relation of $x$ to other elements of $A$ must be known also. Another example of this is given in the algebra (cf. examples 9.2, 9.8) whose basis is

$$
\cdots, \quad x^{-2}, \quad x^{-1}, \quad e \cdots-x^{0}, \quad r, \quad x^{2}, \quad \cdots
$$

and in which it is assumed that every element of the form $\sum_{-n}^{\infty} \xi_{i} x^{i}$ exists for finite values of $n$. In this algebra the subalgebra of all elements of the form $\sum_{1}^{\infty} \xi_{i} x^{i}$ does not seem to be distinguishable from the one discussed above, although in the complete algebra $x$ has the inverse $x^{-1}$.

Instead of attempting to resolve these difficulties by a discussion of the nature of a basis in general, we shall be content for the present to introduce postulates which would most probably appear as theorems if a different mode of attack on the problem were used.

Postulate 5.4. If $e$ is a primitive idempotent clement of $A$, $x$ an element of $e$ Ae which does not have an inverse with respect to $e$, and $y=e-x$, then either.

$$
r^{\prime}+x^{2}+x^{3}+\cdots \quad \text { or } \quad y+y^{2}+y^{3}+\cdots
$$

exists as an element of $A$.

It follows from this postulate that y has an inverse with respect to $e$, namely $z=e+x+x^{2}+\cdots$. For if $z$ exists, evidently $y z=e$; and, if $z$ does not exist, by our postulate $u=-e+y+y^{2}+\cdots$ exists, which is impossible as it would then be an inverse of $x$ with respect to $e$. 
An element of an algebra $A$ which does not have an inverse with respect to any idempotent element of $A$ will be said to be singular* in $A$. With reference to such elements we have the following theorems.

Theorem 5.1. If $e$ is a primitive idempotent element, every element $x$ of o A $e$ which is singular in $e A e$ is also singular in $A$.

If $x$ is not singular in $A$, there is an element $y$ such that $e_{1}=x y$ is idempotent, where, since $e_{1}=x y e_{1}$, we may assume $y e_{1} \cdots y$. Since $e x=x$, it follows that $e e_{1}=e_{1} ;$ also, if $e_{2}=\cdots e_{1} e$, then $e_{2}=e_{1} e$ and

$$
e_{2}^{2}=e e_{1} e e_{1} e=e e_{1}^{2} e=e e_{1} e \cdots e_{2} \text {. }
$$

Hence, since $e_{2}<e A e$ and $e$ is primitive, either $e_{2}=0$ or $e_{2}=e$. If $e_{2}=0$, then $e_{1}=e_{1}^{2}=e_{1} e e_{1}=e e_{1} e e_{1}=e_{2} e_{1}=0$; if $e_{2}=e$, then $x y e=e$, contrary to the assumption that $x$ is singular in $e A e$. The theorem is therefore proved.

ThEOREM 5.2. If $e$ is an idempotent element of A, any idempotent element which is primitive in eAe is also primitive in $A$.

Let $e_{1}$ be an idempotent element of $e A e$. If $e_{1}$ is not primitive in $A$, there is a primitive idempotent element $e_{2}$ for which $e_{1} e_{2}=e_{2}=e_{2} e_{1}$, from which it follows that

$$
e_{2}=e_{1} e_{2} e_{1}=e e_{1} e_{2} e_{1} e<e A e .
$$

If, therefore, $e_{1}$ is primitive in $e A e$, it must also be primitive in $A$.

We give now another postulate which includes Postulate 5.4 but is here stated separately as it is not used till Theorem 7.5 is reached, and even there it is not strictly speaking necessary.

Postulate 5.5. If $x$ is singular in $A$, every element of the form $\sum \xi_{n} x^{n}$ exists in $A$.

To prove Postulate 5.4 on this basis we may proceed as follows. If $x<e A e$ and if there is an element $z$ such that $z x$ is idempotent, then $e_{1}=x z e$ is idempotent and commutative with $e$; for, since $e x=x$,

$$
x z e x z e=(x z)^{2} e \cdots x z e .
$$

* Scorza, Rendiconti del Circolo Matematico di Palermo, vol. 45 (1921), p.41, uses the term "exceptional" in much the same sense; but, as his definition implies either a finite basis or that the element is nilpotent, I have thought it necessary to use a different term. D, p. 46, calls exceptional elements "properly nilpotent". 
Since $e$ is primitive, this is impossible unless $e_{1}=e$ or $e_{1}=0$ (in which case it is not strictly speaking idempotent). If $e_{1}=e$, then $z e$ is an inverse of $x$ with respect to $e$; if $e_{1}=0$, then

$$
0=x z e x z=(x z)^{2}=x z,
$$

which is impossible since $x z$ is idempotent. Hence $x$ is either singular or has an inverse with respect to $e$, from which Postulate 5.4 follows immediately.

6. Singular invariant subalgebras. A singular subalgebra $B$ of an algebra $A$ is defined as a subalgebra no element of which has an inverse with respect to any idempotent element of $A$. If $B=A$, then $A$ contains no idempotent element and is said to be singular in itself; if, on the other hand, $A$ does contain an idempotent element, we shall say that it is nonsingular. For example, a nilpotent subalgebra is singular in any algebra in which it is invariant; or again, the algebra whose basis is $x, x^{2}, x^{3}, \ldots$ is not singular in the algebra $\cdots, x^{-1}, e=x^{0}, x, x^{2}, \cdots$ while it is singular in the subalgebra $e, x, x^{2}, \ldots$.

A semi-simple algebra is one which is non-singular and which possesses no singular invariant subalgebra.

We shall now show that singular invariant subalgebras have, in the main, the properties possessed by nilpotent invariant subalgebras in the case of algebras that have a finite basis.

THEOREM 6.1. If an element $x$ is singular in an invariant stibalgebra $B$ of $A$, it is also singular in $A$.

For, if $x y=e$ were idempotent, then $e<B$ since $x<B$ and $B$ is invariant.

THEOREM 6.2. If an invariant subalgebra $B$ of an algebra $A$ contains no idempotent element, it is singular; and if it contains an idempotent element, it also possesses a primitive idempotent element of $A$.

The first part of this theorem follows immediately from the proof of Theorem 6.1. If $B$ contains an idempotent element, then by Postulate 5.1 there is an idempotent element $e$ which is primitive in $B$. If $e$ is not primitive in $A$, there is a primitive idempotent element $e_{1} \neq e$ in $A$ such that $e e_{1}=e_{1}$. Since $e$ lies in $B$, which is invariant, it follows that $e_{1}<B$, which is impossible since $e$ is primitive in $B$. Hence $e$ must be primitive in $A$ as well as in $B$.

THEOREM 6.3. If $e$ is an idempotent element of $A$, any element of eAe which is singular in eAe is also singular in $A$.

For, if $x<e A e$ and $x y=e^{\prime}$, where $e^{\prime}$ is idempotent, then $e e^{\prime}=e x y$ $=x y=e^{\prime}$ and $x y e=e e^{\prime} e=e^{\prime \prime}<e A e ;$ also $e^{\prime \prime 2}=e e^{\prime} e e^{\prime} e=e e^{\prime} e=e^{\prime \prime}$ 
and $e^{\prime \prime} \neq 0$ since $e^{\prime \prime} e^{\prime}=\left(e e^{\prime}\right)^{2}=e^{\prime}$, so that $e^{\prime \prime}$ is an idempotent element of $e A e$ relative to which $x$ has an inverse.

THeorem 6.4. The totality of elements which are singular in $A$ form a singular invariant subalgebra $S$ of $A$ which contains every singular invariant subalgebra of $A$.

This theorem is proved as follows. If $x$ is singular, so is also $y x$; for, if it is not singular, there is an element $z$ for which $z y x=e$ is idempotent, which contradicts the assumption that $x$ is singular. Hence, if $B$ is the totality of elements which are singular in $A, A x$ and $x A$ are contained in $B$, which is therefore closed under the operation of multiplication and has invariantive properties. We have then only to prove that $B$ is closed under the operation of addition as it is clear that it will then be a singular invariant subalgebra.

Let $x_{1}$ and $x_{2}$ be elements of $B$ and suppose, if possible, that $x_{1}+x_{2}$ has an inverse $y$, say $x_{1} y+x_{2} y=e$ where $y e=e$ and $e$ is idempotent; since $x_{1} y<x_{1} A<B, x_{2} y<x_{2} A<B$, we may obviously assume that $x_{1}+x_{2}=e, e x_{1}=x_{1}=x_{1} e, e x_{2}=x_{2}=x_{2} e$. If $e$ is not primitive, there exists a primitive idempotent element $e_{1}$ for which $e e_{1}=e_{1}=e_{1} e$, so that $x_{1} e_{1}+x_{2} e_{1}=e_{1}$; we may therefore assume that $e$ is primitive. This is, however, impossible in view of Postulate 5.4, and hence $B$ is closed under addition.*

THEOREM 6.5. If $S$ is the maximal singular invariant subalgebra of a non-singular algebra $A, A-S$ is semi-simple.

Let

$$
A=B+S, \quad B-S=0 .
$$

$A-S$ is non-singular since we may choose $B$ so as to contain at least one of the idempotent elements that exist in $A$. If, then, $A-S$ has a proper invariant singular subalgebra $T$, there is in $B$ a complex $T_{1}$ that contains no element $x$ for which $x^{2} \equiv x(\bmod S)$ and for which

$$
B T_{1} \leqq T_{1}, \quad T_{1} B \leqq T_{1}
$$

$(\bmod S)$

It follows that $T_{1}+S$ is, in $A$, a proper invariant subalgebra which contains $S$. Since $S$ is maximal, $T_{1}+S$ must contain an idempotent

${ }^{*}$ In W, p. 91, Theorem 15 , the statement that the totality $B$ forms an invariant subalgebra was omitted. My attention was called to the need of this addition to the theorem by Professor L. E. Dickson in 1914 and the proof given here is essentially the one made at that time. When $A$ has a finite basis, Postulate 5.4 is superfluous since the series $x+x^{2}+x^{3}+\cdots$ terminates when $x$ is nilpotent. A different proof is given by Scorza, loc. cit., p. 42 . 
element $e$, which we may take to be primitive in view of Theorem 6.2. We have therefore $e=x+y$, where $x<T_{1}$ and $y<S$; and $x \neq 0$, since $e \Varangle S$. This gives $x^{2} \equiv x(\bmod S)$, whereas $T_{1}$ contains no such element; the theorem then follows immediately.

THEOREM 6.6. If $N$ is a maximal nilpotent invariant subalgebra of $A$ every nilpotent invariant subalgebra of $A$ is contained in $N$.

This theorem is proved in much the same way as when $A$ has a finite basis. Let $N_{1}$ be any nilpotent invariant subalgebra of $A$ other than $N ; N+N_{1}$ is then also invariant. If $N_{2}=N \frown N_{1}$, we have

$$
\left(N+N_{1}\right)^{n} \leqq N^{n}+N_{1}^{n}+N_{2}
$$

for every positive integer $n$. If the indices of $N$ and $N_{1}$ are finite, it follows immediately that $N+N_{1}$ is nilpotent. If either index is transfinite, we have

$$
\left(N+N_{1}\right)^{\omega} \leqq N^{\omega}+N_{1}^{\omega}+N_{2}=N_{3},
$$

from which we derive in the same way

$$
\left(N+N_{1}\right)^{\omega^{2}} \leqq N_{3}^{\omega} \leqq N^{\omega^{2}}+N_{1}^{\omega^{2}}+N_{2}^{r}=N_{4},
$$

and so on. If then $\nu$ is the greater of the indices of $N$ and $N_{1}$, it follows that $\left(N+N_{1}\right)^{\nu} \leqq N_{2}$, which is nilpotent. Since $N$ is maximal it follows that $N_{1}<N$.

If $N$ is a nilpotent invariant subalgebra which is maximal with respect to the property of having a finite index (so that $N$ is possibly contained in some nilpotent invariant subalgebra whose index is transfinite) the same proof shows that every nilpotent invariant subalgebra whose index is finite is contained in $N$; for if the index of $N$ is finite, so is also the index of each of its subalgebras and, in particular, the index of $N_{2}$ in the above proof is finite.

THeOREM 6.7. Every algebra $A$ which does not have a modulus either has a singular invariant subalgebra or is itself singular.

Let $e$ be a principal idempotent element of $A$ and let

$$
A=A_{00}+A_{10}+A_{01}+A_{11}
$$

be the Peirce decomposition of $A$ relative to $e$. 
Suppose in the first place that $A_{00} \neq 0$. If some element of $A_{00}$ is not singular in $A, A_{00} x$ must contain an idempotent element for some $x<A$. But $A_{00} x<A_{00}+A_{01}$; hence we must have an idempotent element of the form $x_{00}+x_{01}$, where $x_{00}<A_{00}, x_{01}<A_{01}$. This gives

$$
x_{00}+x_{01}=\left(x_{00}+x_{01}\right)^{2}=x_{00}^{2}+x_{00} x_{01} \text {. }
$$

Now $x_{00}^{2}<A_{00}, x_{00} x_{01}<A_{01}$ and $A_{00}-A_{01}=0$; hence $x_{00}=x_{00}^{2}$, and, seeing that $A_{00}$ contains no idempotent element, it follows that $x_{00}=0$ and therefore $x_{01}=x_{00} x_{01}=0$. The elements of $A_{00}$ are therefore singular in $A$.

If $A_{00}=0$, then $A_{10}+A_{01} \neq 0$ since $A$ has by supposition no modulus. For any $x<A$,

$$
\left(A_{10}+A_{01}\right) x<A_{01}+A_{10} A_{01},
$$

and hence, if $A_{10}+A_{01}$ is not composed entirely of singular elements, there must be an idempotent element of the form $x_{01}+x_{11}$ where $x_{01}<A_{01}$, $x_{11}<A_{10} A_{01}$ and

$x_{11}^{2}<A_{10} A_{01} A_{10} A_{01}<A_{10} A_{00} A_{01}=0, \quad x_{01} x_{11}<x_{01} A_{10} A_{01}<A_{00} A_{01}=0$.

This gives

$$
x_{01}+x_{11}=\left(x_{01}+x_{11}\right)^{2}=0,
$$

so that there is no idempotent element in $A_{10}+A_{01}$.

Hence in every case $A$ contains singular elements and by Theorem 6.4 also a singular invariant subalgebra.

7. Simple algebras. The discussion of the structure of a simple algebra parallels closely the corresponding theory for algebras with a finite basis.

THEOREM 7.1. A simple algebra, which is not singular, possesses a modulus.

This theorem is an immediate consequence of Theorem 6.6 but, because the proof given there depends on Postulate 5.4, it seems worth while to give an independent demonstration. Since an algebra $A$ which is not singular possesses a principal idempotent element $e$, we may express $A$ in Peirce's form

where

$$
A=A_{00}+A_{10}+A_{01}+A_{11}
$$

If we set

$$
\begin{aligned}
& e A_{1 j}=A_{1 j}, \quad e A_{0 j}=0 \\
& A_{j 1} e=A_{j 1}, \quad A_{j 0} e=0 .
\end{aligned}
$$

$$
B_{1}=A_{00}+A_{10}, \quad B_{2}=A_{00}+A_{01},
$$


then

$$
\begin{aligned}
B_{1} A & =B_{1} B_{2}=A B_{2}, \\
A B_{1} B_{2} & =A A B_{2} \leqq A B_{2}=B_{1} B_{2} \\
B_{1} B_{2} A & =B_{1} A A \leqq B_{1} A=B_{1} B_{2}
\end{aligned}
$$

and therefore, since $A$ is simple, we must have $B_{1} B_{2}=0$. This gives

$$
\begin{aligned}
\left(B_{1}+B_{2}\right)^{2} & =B_{1}^{2}+B_{2}^{2}+B_{1} B_{2}+B_{2} B_{1} \leqq A_{00}, \\
A\left(B_{1}+B_{2}\right) & =A B_{1}+A B_{2}=A B_{1} \leqq B_{1} \leqq B_{1}+B_{2}, \\
\left(B_{1}+B_{2}\right) A & =B_{1} A+B_{2} A=B_{2} A \leqq B_{2} \leqq B_{1}+B_{2} .
\end{aligned}
$$

But $A$ is simple; hence $B_{1}+B_{2}=0$, that is, $A=A_{11}$, which proves the theorem.

TheOREM 7.2. 'If $e$ is an idempotent element of a simple algebra $A, e A e$ is simple.

For, if $B$ is a proper invariant subalgebra of $e A e$,

$$
e A B e=e A e \cdot B \cdot e A e \leqq B<A
$$

therefore $A B A<A$ is a proper invariant* subalgebra of $A$. This is impossible since $A$ is simple.

If $e$ is primitive, it follows from Theorem 6.3 that every element of $e A e$ has an inverse relative to $e$; such an algebra is called a division algebra.

Let $e_{t}, t$ in a range $G$, be a complete primitive complementary set for the simple algebra $A$; then $e=\sum_{G} e_{t}$ is the modulus of $A$ and, if $A_{r s}=e_{r} A e_{s}$, the Peirce form of $A$ is $\sum \sum A_{r s}$. Since $A$ is simple $A e_{s} A=A$, as otherwise it would be an invariant subalgebra of $A$; hence, multiplyingt on the left by $e_{r}$ and on the right by $e_{t}$, we have

$$
A_{r s} A_{s t}=A_{r t}
$$

We shall now show that, if $x_{r s}$ and $x_{s t}$ are any elements, different from 0 , of $A_{r s}$ and $A_{s t}$ respectively, then $x_{r s} x_{s t} \neq 0$. Since $A$ is simple and $e_{t}$ is primitive, $A_{t t}$ is a division algebra for every $t$. Suppose that $x_{r s} x_{s t}=0$;

* Cf. Scorza, loc. cit., p. 15.

$\dagger$ Cf. Scorza, loc. cit., p. 76. It is not necessary here that the $e_{t}$ should be primitive. 
then $x_{r s} x_{s t} A_{t s}=0$. But $x_{s t} A_{t s} \leqq A_{s s}$, which is a division algebra, and hence, for any $y_{t s}<A_{t s}$ such that $x_{s t} y_{t s} \neq 0$, there is a $y_{s s}<A_{s s}$ for which $x_{s t} y_{t s} y_{s s}=e_{s}$. Hence, as $x_{r s} \neq 0, x_{r s} x_{s t} A_{t s}=0$ entails $x_{s t} A_{t s}=0$. It follows for every $x_{t s}<A_{t s}$ that $x_{s t} x_{t s}=0$, and therefore $x_{t s} A_{s t}=0$ by a repetition of the same argument. But $x_{t s}$ is any element of $A_{t s}$ so that our supposition that $x_{r s} x_{s t}=0$ has led to $A_{t s} A_{s t}=0$, which contradicts (7.1); hence $x_{r s} x_{s t} \neq 0$ unless one of the factors is 0 .

Using the same reasoning as above, only multiplying on the right instead of on the left, we see that there is an element $y_{s r}<A_{s r}$ for which $y_{s r} x_{r s}=e_{s}$; also, if we set $x_{r r}=x_{r s} y_{s r}$, then

$$
x_{r r}^{2}=x_{r s} \cdot y_{s r} x_{r s} \cdot y_{s r}=x_{r s} e_{s} y_{s r}=x_{r r}
$$

and therefore $x_{r r}=e_{r}$, seeing that the latter is primitive. Further, since

it follows that

$$
A_{s t}=y_{s r} x_{r s} A_{s t} \leqq y_{s r} A_{r t} \leqq A_{s t}
$$

and

$$
A_{s t}=y_{s r} A_{r t}
$$

$$
x_{r s} A_{s t}=x_{r s} y_{s r} A_{r t}=A_{r t} .
$$

We shall now show that it is possible to find a set of elements $e_{r s}\left(r, s\right.$ in $\left.G, e_{r s}<A_{r s}\right)$ such that $e_{r s} e_{s t}=e_{r t}, e_{r s} e_{t u}=0(s \neq t)$, and $e=\sum e_{t t}$. Set $e_{t t}=e_{t}$ and let $e_{p t}$ be any non-zero element of $A_{p t}, p$ being a fixed and $t$ a variable element* of $G$. We have already shown that there then exist elements $e_{t p}$ such that $e_{t p} e_{p t}=e_{t}$ and $e_{p t} e_{t p}=e_{p p}$, and, if we set in general $e_{s t}=e_{s p} e_{p t}$, it is readily seen that the elements so defined have the required property. We are now ready to prove the following fundamental theorem.

THEOREM 7.3. Every simple algebra with a modulus can be expressed as the direct product of a division algebra and a simple matric algebra.

Since $A_{s t}=e_{s p} A_{p p} e_{p t}$, there is a $(1,1)$-correspondence between the elements of each $A_{s t}$ and those of a fixed $A_{p p}$, which, as we have seen, is a division algebra. The theorem then follows exactly as in the case of algebras with a finite basis.t We have also the converse theorem.

THEOREM 7.4. The direct product $A$ of a simple matric algebra $B$ and $a$ division algebra $C$ is simple; and any element of $A$ which is commutative with every element of $A$ is an element of $C$.

* Cf. Scorza, loc. cit., p. 78.

$\dagger$ Cf. W, p. $98 ;$ D, p. 76. 
The proof is the same as for algebras with a finite basis.*

THeOREM 7.5. If $S$ is the maximal singular invariant subalgebra of an algebra $A$ which possesses a modulus, and if $A-S$ is simple, $A$ can be expressed as the divect product of a simple matric algebra and an algebra whose modulus is its only idempotent element.

We shall not in the first instance assume that $A$ has a modulus but only that $A-S$ is simplet and $A \neq S$. Let $e_{t}, t$ in $G$, be a complete primitive complementary set of idempotent elements of $A$; the elements of this set are linearly independent modulo $S$ since, were $\sum \xi_{t} e_{t} \equiv 0$ $(\bmod S)$, we should have

$$
0 \equiv e_{p} \sum \xi_{t} e_{t} \equiv \xi_{p} e_{p}
$$

whereas $e_{p} \Varangle S$ for any $p$. It follows from the proof of Theorem 7.3 (taking into account the second footnote of page 414$)$ that $A_{r s} A_{s t} \equiv A_{r t}(\bmod S)$. Now $A_{r s} A_{s r}$ is an invariant subalgebra of $A_{r r}$ which is not singular seeing that it is not congruent to 0 modulo $S$; and since $e_{r}$ is primitive, it follows from Theorem 6.4 that any proper invariant subalgebra of $A_{r r}$ is necessarily singular; hence $A_{r s} A_{s r}=A_{r r}$. We have also $A_{r r} A_{r s} \geqq A_{r s}$ because $A_{r r}$ contains $e_{r}$ and, since $A_{r s} \geqq A_{r t} A_{t s}$,

$$
A_{r s} A_{s t} \geqq A_{r t} A_{t s} A_{s t}=A_{r t} A_{t t} \geqq A_{r t}
$$

and also $A_{r s} A_{s t} \leqq A_{r t}$; hence $A_{r s} A_{s t}=A_{r t}$.

We must now prove that $x_{r s} A_{s r}=A_{r r}$ when $x_{r s}$ is an element of $A_{r s}$ which does not belong to $S$. Now $x_{r s} A_{s r} \neq 0(\bmod S)$; for, if this were so, then $x_{r s} A_{s s}=x_{r s} A_{s r} A_{r s} \equiv 0(\bmod S)$, which is impossible since $x_{r s}=x_{r s} e_{s}<x_{r s} A_{s s} \Varangle S$ so that $x_{r s} A_{s r}$ contains non-singular elements of $A$ and, by Theorem 6.3, also elements which are not singular in $A_{r r}$. Hence if $x$ is any non-singular element of $x_{r s} A_{s r}$, there is an element $y$ of $A_{r r}$ such $x y=e_{r}$, so that

$$
e_{r}=x y<x_{r s} A_{s r} A_{r r} \leqq x_{r s} A_{s r}
$$

that is, to any $x_{r s} \Varangle S$, there is an element $x_{s r}<A_{s r}$ such that $x_{r s} x_{s r}=e_{r}$. It then follows as in the proof of Theorem 7.3 that $x_{r s} A_{s t}=A_{r t}$, that there exists a simple matric algebra $e_{r s}, r, s$ in $G$, and that $\sum \sum A_{r s}$ is

* Cf. W, p. 99; D, p. 79.

$\dagger$ It then follows from Theorem 7.1 that $A-S$ has a modulus. 
the direct product of this simple matric algebra and any $A_{w}$. If we now add the condition that $A$ has a modulus so that $A=\sum \sum A_{r s}$, the proof of the theorem is complete.

The above discussion renders it probable that an idempotent element which is primitive in $A$ corresponds to a primitive idempotent element in $A-S$; and in fact this can be shown by a somewhat roundabout argument. If, however, Postulate 5.5 is assumed, the proof is more direct and also contains some points of intrinsic interest.

If $x^{2}-x=y \equiv 0(\bmod S)(x \Varangle S)$ and we set $z=f(y)+g(y) x$, we readily find that a formal solution of the equation $z^{2}=z$ is given by*

$$
\begin{aligned}
z & =\frac{2 x-1}{2 \sqrt{1+4 y}}+\frac{1}{2} \\
& =x-2 x\left(y-6 y^{2}+\cdots\right)+y-6 y^{2}+\cdots \equiv x \quad(\bmod S),
\end{aligned}
$$

where Postulate 5.5 is required in order that the series used should exist as elaments of $A$. If $z$ is primitive in $A$ but $x$ does not correspond to a primitive idempotent element in $A-S$, we may write $x=x_{1}+x_{2}$ where

$$
x_{1}^{2}-x_{1} \equiv x_{2}^{2}-x_{2} \equiv x_{1} x_{2} \equiv x_{2} x_{1} \equiv 0 \quad(\bmod S),
$$

and we may, without loss of generality, suppose $x_{1}$ modified as above so that $x_{1}^{2}=x_{1}$, since this still leaves $x \equiv z(\bmod S)$. If $x_{1} x_{2}=y_{12}, x_{2} x_{1}=y_{21}$, we have $y_{12}=x_{1} x_{2}=x_{1}^{2} x_{2}=x_{1} y_{12}$ and therefore $x_{1}\left(x_{2}-y_{12}\right)=0$; we may therefore suppose $y_{12}=0$ and, since then $x_{1}\left(x_{2}^{2}-x_{2}\right)=0$, we may modify $x_{2}$ as before so that it is idempotent and still keep $x_{1} x_{2}=0 . x_{3}==x_{2}-x_{2} x_{1}$ is then idempotent and $x_{1} x_{3}=0=x_{3} x_{1}$. We shall therefore assume $x_{1} x_{2}=0=x_{2} x_{1}, x_{1}^{2}=x_{1}, x_{2}^{2}=x_{2}$ and $z=x_{1}+x_{2}+y$ where $y<S$. This gives

$$
z=-x_{1}+x_{2}, \quad z x_{1} z=x_{1}, \quad z x_{2} z=x_{2} \quad(\bmod S)
$$

and hence, seeing that $z$ is primitive in $A$, there exist elements $w_{1}$ and $w_{2}$ such that

$$
x_{1} w_{1} \equiv z, \quad x_{2} w_{2} \equiv z \quad(\bmod S)
$$

The first of these congruences gives $x_{2} z \equiv 0(\bmod S)$ and the second

${ }^{*}$ If $2 \equiv 0$ in the field, we may set $z=x+y+y^{2}+y^{4}+y^{8}+\cdots$. 
$x_{2} z \equiv z$; the supposition that the element in $A-S$ corresponding to $z$ is not primitive has therefore led to a contradiction.

The methods which are used in the theory of algebras with a finite basis to show that a simple algebra cannot be singular except in the trivial case of the algebra that consists of one unit $x$ for which $x^{2}=0$, depend on induction and therefore cannot be extended directly when a finite basis is wanting. We can however apply these methods to prove the following theorem.

THEOREM 7.6. A simple commutative algebra is either a division algebra, or is the algebra of one unit $x$ for which $x^{2}=0$.

If $x$ is any non-zero element, we must have $A x=A$, since otherwise, if $A x \neq 0$, it is a proper invariant subalgebra of $A$ and, if $A x=0,\{x\} \equiv(x)$ is a proper invariant subalgebra of $A$, unless of course $A \equiv(x)$. Moreover, if $z$ is any other non-zero element, we cannot have $z x=0$ since, were this so, we should have

$$
A=A z, \quad A=A x=A z x=0 .
$$

But, if $A x=A$, there must be an element $e$ such that $e x=x$; this gives $\left(e^{2}-e\right) x=0$ and therefore $e^{2}-e=0$ so that $e$ is idempotent. We have shown that the product of two elements can only vanish if one of them is zero; hence $A$ is a division algebra with $e$ as modulus.

8. Reducibility. The principal theorems regarding the uniqueness of the expression of an algebra as a direct sum which are true of algebras with a tinite basis also hold when no finite basis exists. Before showing this however we must first prove the following theorem, which is trivial when the basis is finite.

The0REm 8.1. Every reducible algebra $A$ that has a modulus possesses an irreducible reduced part.

Let $e_{t}, t$ in $G$, be a complete primitive set of idempotent elements. If $A_{r s}=e_{r} A e_{s}$, then no $A_{r r}$ is 0 since $A_{r r}$ contains $e_{r}$. If for some fixed $r$ every $A_{r s}$ and $A_{s r}(s \neq r)$ is 0 , then evidently $A$ is reducible with $A_{r r}$ as one reduced part; and $A_{r r}$ is irreducible since the only idempotent element it contains is its modulus $e_{r}$, while Theorem 4.4 requires that each part of a reducible algebra with a modulus shall also have a modulus. We may suppose therefore that $A_{r s}+A_{s r} \neq 0$ for some $s \neq r$; then that $A_{s s_{1}}+A_{s_{1}} \neq 0$ for some $s_{1} \neq s$, and so on. We define in this way a subset $H$ of the range $G$ such that (i) if $s$ is a value of $t$ in $H$, there are a finite number of values of $t$ in $H$, say $s_{1}, s_{2}, \cdots, s_{n}$, for which $A_{r s_{1}}+A_{s_{1} r}, A_{s_{1} s_{2}}+A_{s_{2} s_{1}}, \cdots, A_{s_{n} s}+A_{s s_{n}}$ are all different from 0 , and (ii) all values of $s$ which may be reached in this manner from $r$ in a finite number of steps are contained in $H$. 
Let $K$ be the complement of $H$ in $G$ and set

$$
e_{1}=\sum_{H} e_{t}, \quad e_{2}=\sum_{K} e_{t}
$$

If $e_{1} A e_{2} \neq 0$, there must be some $p<H$ and $q<K$ for which $A_{p q} \neq 0$; but $p$ can be reached from $r$ in a finite number of steps and hence $q$ also, in contradiction to the definition of $H$ and $K$. Hence $e_{1} A e_{2}=0$ and similarly $e_{2} A e_{1}=0$, so that $A$ is reducible. Moreover $e_{1} A e_{1}=A^{11}$ is irreducible; for if $A^{11}=B \oplus C$, then $e_{r}$, being primitive, must lie in either $B$ or $C$, say $B$, and, if $e_{s}$ is any one of the original set of idempotent elements which belongs* to $C, s$ cannot be reached from $r$ by a finite number of the steps used in the definition of $H$, since, if $e_{p}<B, e_{q}<C$, then $A_{p q}$ and $A_{q p}$ are both zero.t The proof of the theorem is therefore complete.

We are now ready to prove the theorems referred to at the beginning of the section.

Theorem 8.2. If an algebra $A$ which has a modulus is expressed in two ways as the direct sum of irreducible parts, these two expressions differ only in the order in which the constituent parts occur and both contain every irreducible reduced part of $\mathbf{A}$.

By Theorem 8.1 $A$ possesses irreducible reduced parts; let $A_{t}, t$ in a range $G$, denote these parts and set $B=\sum_{G} A_{t} ; B$ is then an invariant subalgebra of $A$ since by Postulate $5.3 A B=\sum A A_{t}=\sum A_{t}=B$, and similarly $B A=B$. By Theorem 4.4 every $A_{t}$ has a modulus $e_{t}$ and, by Theorem 4.6, $A_{r} A_{s}=0(r \neq s)$; also, by Postulate 5.2, $e^{\prime}=\sum e_{t}$ exists, and it is evidently the modulus of $B$. Hence, by Theorem 4.3, $B$ is a reduced part of $A$, say $A=B \oplus C$. But, by Theorem 4.4, $C$ has a modulus and therefore it is either itself an irreducible reduced part of $A$ or it contains such a part. This is impossible since $B-C=0$, and therefore $C=0$, whence $B=A$.

If now $A=\sum B_{s}$ is any expression of $A$ as the direct sum of irreducible parts, then $A_{t}=e_{t} A e_{t}=\sum_{s} e_{t} B_{s} e_{t}$. If $e_{t} B_{s} e_{t} \neq 0$, then by Theorem 4.3 $e_{t} B_{s} e_{t}=B_{s}$, since it is an invariant subalgebra of $B_{s}$ which has a modulus and $B_{s}$ is irreducible; also, since $e_{t}<A_{t}$, which is invariant in $A$, it follows in the same way that $e_{t} B_{s} e_{t}=A_{t}$. Hence $A_{t}=B_{s}$, that is, every $A_{t}$ occurs in the set $B_{s}$, and, since the set $A_{t}$ contains every irreducible reduced part of $A$, the set $B_{s}$ is only a rearrangement of it.

\footnotetext{
${ }^{*}$ If $e_{e}$ is a primitive idempotent element, $e_{1} e_{s} e_{1}=e^{\prime}$ and $e_{2} e_{s} e_{2}=e^{\prime \prime}$ are idempotent if not zero; but $e_{\mathrm{a}}=e^{\prime}+e^{\prime \prime}, e^{\prime} e^{\prime \prime}=0=e^{\prime \prime} e^{\prime}$ and $e_{\mathrm{a}}$ is primitive; hence one of $e^{\prime}, e^{\prime \prime}$ is 0 . Any primitive idempotent element therefore belongs to one or other of $B$ and $C$.

f For instance, $A_{p q}=e_{p} A e_{q}=e_{p} e_{1} A e_{1} e_{q}=0$.
} 
TheOREm 8.3. Every algebra A which has a modulus either has no invariant subalgebra which has a modulus or can be expressed uniquely as the direct sum of such an algebra and an algebra which has a modulus.

Let $B^{\prime}$ be an invariant subalgebra of $A$ which has a modulus; then by Theorem 4.3, $A=B^{\prime} \oplus C^{\prime}$, and therefore $A$ possesses at least one irreducible reduced part which has a modulus. As in the previous theorem, the algebra $B$ which is the sum of all the irreducible reduced parts of $A$ that have a modulus is the direct sum of these parts and has a modulus. Hence, by Theorem 4.3, $A=B \oplus C$. Here $C$ has no modulus, since $A$ has none, and it has no irreducible reduced part with a modulus, since any such part is also an irreducible part of $A$ and so belongs to $B$, whereas $B-C=0$. By Theorem 4.3 and its corollary, $C$ has no invariant subalgebra with a modulus and is unique. The theorem is therefore proved.

THEOREM 8.4. An algebra which has no modulus and no invariant subalgebra with a modulus becomes irreducible if a modulus is added to it.

For if $A^{*}$ denote the algebra obtained by adding a modulus $e$ to $A$, and if $A^{*}=B \oplus C$, then each of $B$ and $C$ has a modulus, say $e_{1}$ and $e_{2}$. Now $e_{1}=x+\xi e, e_{2}=y+\eta e$, where $x, y<A$ and $\xi, \eta$ are scalars. But $e_{1} e_{2}=0$ and therefore $\xi \eta=0$. If, say, $\xi=0$, then $e_{1}<A$ and therefore also $B=e_{1} A^{*} e_{1}<A$; and this is impossible, as $B$ would then be a reduced part of $A$ with a modulus.

9. Illustrative examples. We shall now give a number of examples some of which are given to illustrate the theory of the preceding sections and others because of their intrinsic interest. In most of these examples we shall denote the basi of $A$ by $x_{t}$ where the variable $t$ runs through a range $G ; x=\sum_{G} \xi_{t} x_{t}, y=\sum_{G} \eta_{t} x_{t}$, etc., will denote general elements of $A$ and $\xi(t), \eta(t)$, etc., the corresponding functions of $t$. The elements $x_{t}$ are linearly independent unless otherwise stated.

Example 9.1. Let

$$
x_{t}^{2}=x_{t}, \quad x_{t} x_{s}=0
$$

Then $x+y=\sum\left(\xi_{t}+\eta_{t}\right) x_{t}$ and $x y=\sum \xi_{t} \eta_{t} x_{t}$, and, in the notation of $\S 2, \xi(\alpha) \times \eta(\alpha)=\xi(\alpha) \eta(\alpha)$. The functional product in this case therefore corresponds to ordinary multiplication.

Example 9.2. Let

$$
x_{t} x_{s}=x_{t+s}
$$

We shall call the corresponding algebra the power algebra since this is what $A$ becomes when $G$ is the set of positive integers. Here we have

$$
x y=\sum_{G(t)} \sum_{G(s)} \xi_{s} \eta_{t-s} x_{t},
$$


or

$$
\xi(t) \times \eta(t)=\sum_{G(s)} \xi(s) \eta(t-s) .
$$

(9.21) Let $G$ be the set of positive integers; the order of $A$ is then $x$. If $a=x_{1}$, then $x_{t}==a^{t}$, so that this algebra is the algebra of one indeterminate, that is, it uses the Grassmann indeterminate or general product. If 0 is included in $G, x_{0}$ is the modulus and the algebra is then equal to $\left\{x_{0}, a\right\} ;\{a\}$ is the maximal invariant subalgebra.

In this algebra the summation sign $\Sigma$ indicates ordinary algebraic summation, but no question of convergence is involved so long as the given basis is used.

(9.22) Let $G$ be the set of positive and negative integers and 0 . Here a distinction must be made between the case in which $\sum$ refers to sums with a finite number of terms only and that in which infinite sums are allowed. In the latter case $A$ is, in some sense at least, equivalent to (9.21); for, if $e=x_{0}, b=e+x_{1}=e+a$, then

and so on.

$$
a=e-b, \quad a^{-1}=e+b+b^{2}+\cdots,
$$

(9.23) Let $G$ be the interval $\alpha<t<\infty$ in the real continuum or, alternatively, $\alpha \leqq t<\infty$. As before we may set $x_{t}=a^{t}$; the algebra has then a modulus only if $G$ includes $t=0$. If certain integrability conditions are satisfied by the functions involved, $\sum_{G}$ may be interpreted as ordinary integration. For instance, if $\alpha=0$, we may set*

$$
\xi(t) \times \eta(t)=\int_{0}^{t} \xi(s) \eta(t-s) d s=t \int_{0}^{1} \xi(t s) \eta[t(1-s)] d s .
$$

It is easily shown that this product is associative.

(9.24) We may take for $G$ any aggregate of sets of points such that the logical sum of any two sets is also a member of $G$.

Example 9.3. An algebra closely allied to the preceding one is given by

$$
\begin{aligned}
x_{t} x_{s} & =x_{t s}, \\
x y & =\sum_{t} \sum_{s} \xi_{s} \eta_{t / s} x_{t},
\end{aligned}
$$

or

$$
\boldsymbol{\xi}(t) \times \eta(t)=\sum_{G} \boldsymbol{\xi}(s) \eta\left(\frac{t}{s}\right)
$$

\footnotetext{
* This product and the one given in (9.321) below are of course well known, usually
} with a somewhat more general range than that given here. 
(9.31) If $G$ is the set of positive integers, $A$ is the algebra used by Professor E. T. Bell* in the theory of numbers and called by him a Dirichlet algebra because of its connection with Dirichlet series.

(9.32) If $G$ is the real interval $\alpha \leqq t<\infty$, and $\Sigma$ is interpreted as $\int(\mathrm{)}) \frac{d s}{s}$, we get, when $\alpha=1$,

$$
\xi(t) \times \eta(t)=\int_{1}^{t} \xi(s) \eta\left(\frac{t}{s}\right) \frac{d s}{s}
$$

which may also be written

$$
\log t \int_{0}^{1} \xi\left(t^{s}\right) \eta\left(t^{1-s}\right) \frac{d s}{s} .
$$

Examples (9.2) and (9.3) belong to the special type

$$
x_{s} x_{t}=x_{\varphi(s, t)}, \quad \varphi(r, \varphi(s, t))=\varphi(\varphi(r, s), t) .
$$

When $\varphi$ is properly restricted, it follows from the theory of one-parameter continuous groups that any algebra of this type can be reduced to (9.2) by a change of variable. For instance, if in (9.2) we put $x_{t}^{\prime}=x_{e^{t}}$,

$$
x_{s}^{\prime} x_{t}^{\prime}=x_{e^{s}} x_{e^{t}}=x_{e^{s+t}}=x_{s+t}^{\prime} .
$$

The forms $(9.231,321)$ may be also derived directly from (2.72) by putting

$$
k(r, s, t)=\left\{\begin{array}{l}
1, t=\varphi(r, s), \\
0, t \neq \varphi(r, s),
\end{array}\right.
$$

and interpreting the corresponding integral as a double Stieltjes integral with respect to $k(r, s, t)$.

This type of algebra may be generalized as follows. Let

$$
\varphi_{i}(s ; t) \equiv \varphi_{i}\left(s_{1}, \cdots, s_{n} ; t_{1}, \ldots, t_{n}\right) \quad(i=1,2, \cdots, n)
$$

be a set of functions having the group property

$$
\varphi_{i}(\varphi(r ; s) ; t)=\varphi_{i}(r ; \varphi(s, t)) .
$$

An associative algebra is then defined by

$$
x_{s_{1} s_{2} \cdots s_{n}} x_{t_{1} t_{2} \cdots t_{n}}=x_{\varphi_{1}(s ; t) \varphi_{2}(s ; t) \cdots \varphi_{n}(s ; t)}
$$

* Cf. these Transactions, vol. 25 (1923), p. 135. 
Example 9.4. An example of a non-commutative algebra is given by

$$
\begin{gathered}
x_{s} x_{t}=x_{s+t}+x_{s-t}, \\
\xi(t) \times \eta(t)=\int_{G} \xi(s)[\eta(t-s)+\eta(t+s)] d s .
\end{gathered}
$$

Modifications of this algebra are given by

$$
\begin{aligned}
& x_{s} x_{t}=x_{s+t-k}+x_{s-t+k}, \\
& x_{s} x_{t}=x_{s t}+x_{s / t} .
\end{aligned}
$$

Example 9.5. Corresponding to the ordinary algebra of matrices we have the algebra generated by the units $x_{s t}$, where $s$ and $t$ run through a range $G$ and

$$
x_{s t} x_{p q}=\left\{\begin{array}{l}
x_{s q}, t=p \\
0, \quad t \neq p
\end{array}\right.
$$

This is the simple matric algebra used in $\S 7$. The corresponding functional product is

$$
\xi(s, t) \times \eta(s, t)=\int \xi(s, \tau) \eta(\tau, t) d \tau .
$$

This algebra and its subalgebras have been developed to a considerable extent by writers on the theory of integral equations.

Example 9.6. The Grassmann-Gibbs indeterminate product (the algebra of tensors) forms another important example. Here the units have the

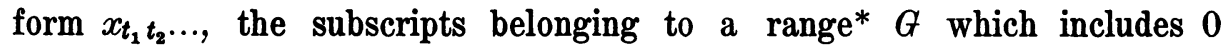
while, if $t_{i}=0$, all subsequent subscripts are also 0 , and the law of combination is

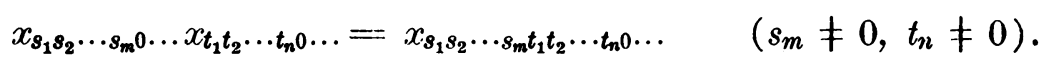

It is usual to add the restriction that the first subscript is not 0 , but, if this is not done, $x_{0} \ldots$ is the modulus of the algebra. The corresponding functional product is

(9.62) $\xi\left(t_{1}, t_{2}, \cdots, t_{m}\right) \times \eta\left(t_{1}, t_{2}, \cdots, t_{n}\right)=\xi\left(t_{1}, t_{2}, \cdots, t_{m}\right) \eta\left(t_{m+1}, \cdots, t_{m+n}\right)$.

* This range is usually the set of positive integers and 0 , but it may of course be taken to be continuous. 
Example 9.7. Let $A$ be the algebra generated by $a, b, c$ where

$$
a b=c, \quad b c=a, \quad c a=b .
$$

If we set $f=a^{2}$, we easily find that $f$ is commutative with every element of $A$ and that

$$
a^{2}=b^{2}=c^{2}=f, \quad b a=f a b, \quad c b=f b c, \quad a c=f c a .
$$

When sums with a finite number of terms alone are allowed, this algebra has no idempotent elements in spite of the fact that $A^{2}=A$, which in the case of algebras with a finite basis always implies the presence of at least one idempotent element. If, on the other hand, infinite sums are allowed, $A$ has exactly four such elements, namely

$$
\frac{1}{2}+\frac{ \pm a \pm b \pm c-(1+f) / 2}{\sqrt{1+14 f+f^{2}}}
$$

where $1 / \sqrt{1+14 f+f^{2}}$ is to be expanded in a series of positive powers of $f$, and the numerical term canceled before interpretation, and the signs are either all + or two - and one + . This algebra may be used to illustrate the Peirce form of $\S 5$ but as the actual expressions are somewhat cumbrous they are not given here.

If $e$ stands for any one of the elements in (9.71), $e A e$ forms a commutative division algebra.

Example 9.8. Let $A$ be the algebra generated by two elements $x$ and $y$ for which

$$
y^{r} \cdot x^{s}=\varepsilon^{r s} x^{s} y^{r}
$$

where $\varepsilon$ is a scalar different from 0 and $1, r, s$ are positive or negative integers or 0 , and $x^{0}=y^{0}=e$ is the modulus of the algebra.

Let $f_{r}(x)(r=0,1, \cdots)$ denote series of the form

$$
x^{n_{r}}\left(\alpha_{0 r} e+\alpha_{1 r} x+\alpha_{2 r} x^{2}+\cdots\right) \quad\left(\alpha_{0 r} \neq 0\right),
$$

where $n_{r}$ is an integer, positive, negative or $0 ; f_{r}(x)$ has a unique inverse of the same type which is obtained by formally inverting the series for $f_{r}$. The algebra is then defined as the set of elements of the form

$$
\sum_{r=0}^{\infty} f_{r}(x) y^{r+n}
$$

where $n$ is an integer which may be negative. The sum and product of two such elements have the same form so that this set does in fact form an algebra. 
We shall now show that every element of the set except 0 has an inverse. We may evidently assume $n=0$, and there is also no loss of generality in taking $f_{0}(x)=e$. If we form the product

$$
\left(e-g_{1} y-g_{2} y^{2}-\cdots\right)\left(e+f_{1} y+f_{2} y^{2}+\cdots\right)
$$

and set $f_{i}^{(r)}=y^{r} f_{i} y^{-r}$, the condition that the result equals $e$ is

$$
g_{1}=f_{1}, \quad g_{2}=f_{2}+g_{1} f_{1}^{\prime}, \quad g_{3}=f_{3}+g_{2} f_{2}^{\prime}+g_{9} f_{1}^{\prime \prime}, \ldots
$$

and hence the $g$ 's are uniquely determined, each has the form of (9.82) and the left hand factor of (9.84) has the form (9.83). Every element therefore has an inverse and hence $A$ is a division algebra.

It should be noted that no question of convergence is involved here, although as a matter of fact the convergence is easily investigated when $|\varepsilon|<1$.

In place of (9.81) we may evidently take

$$
y x=\boldsymbol{\theta}(x) y
$$

where $\theta(x)$ is any function whose iterated powers are known for positive and negative indices. For example we may set

$$
y x=x^{-1} y
$$

which also gives rise to a division algebra under conditions similar to those imposed above. In this algebra $y^{2} x=x y^{2}$, so that every element has the form $f_{1}\left(x, y^{2}\right)+f_{2}\left(x, y^{2}\right) y$.

Example 9.9. Let $A$ be the algebra generated by an element $a$ which satisfies the equation

$$
\varphi(a)=0,
$$

where $\varphi(\xi)$ is an integral function of $\xi$ and the elements of $A$ consist of all integral functions of $a$, that is, of the elements which result from substituting $a$ for $\xi$ in any integral function of $\xi$. The modulus of the algebra then corresponds to the unit 1 of the field and we shall use the same symbol for both.

The function $\varphi(\xi)$ must vanish for some value of $\xi$ in the field of complex numbers; for, if $\varphi(\xi)=\exp \psi(\xi), \psi(\xi)$ integral, then $\exp (-\psi(\xi))$ is also integral so that $\exp (-\psi(a))=b$ is an element of $A$ for which $b \varphi(a)=1$, which contradicts (9.91). If $f(\xi)$ is an integral function of $\xi$ which has no zero in common with $\varphi(\xi)$, the element $f(a)$ of $A$ has 
an inverse; for there always exist integral functions* $A(\xi)$ and $B(\xi)$ such that $A(\xi) \varphi(\xi)+B(\xi) f(\xi)=1$, whence $B(a) f(a)=1$.

Let us suppose now that the roots of $\varphi(\xi)$ are all simple; the MittagLeffler expansion of $1 / \varphi(\xi)$ then has the form

$$
\frac{1}{\varphi(\xi)}=\sum \frac{a_{n}}{\left(\xi-g_{n}\right)}\left(\frac{\xi}{g_{n}}\right)^{m_{n}}+G(\xi)
$$

where $a_{n}=1 / \varphi^{\prime}\left(g_{n}\right)$ and $G(\xi)$ is an integral function and, if we set

$$
\psi_{n}(\xi)=\frac{a_{n}}{\left(\xi-g_{n}\right)}\left(\frac{\xi}{g_{n}}\right)^{m_{n}} \varphi(\xi),
$$

then

$$
1=\sum \psi_{n}(\xi)+\varphi(\xi) G(\xi) .
$$

Since $\psi_{n}(\xi)$ has the form

$$
1+\left(\xi-g_{n}\right) \theta_{1}(\xi)
$$

where $\theta_{1}(\xi)$ is integral, it follows immediately that

$$
\left[\psi_{n}(\xi)\right]^{2}=\psi_{n}(\xi)+\varphi(\xi) \theta_{2}(\xi),
$$

where $\theta_{2}(\xi)$ is integral, and also it is clear from (9.92) that $\psi_{n}(\xi) \psi_{m}(\xi)$ vanishes for every root of $\varphi(\xi)$; hence, if

we have

$$
e_{n}=\psi_{n}(a) \text {, }
$$

and from (9.93)

$$
e_{n}^{2}=e_{n}, \quad e_{m} e_{n}=0 \quad(m \neq n)
$$

$$
\sum e_{n}=1
$$

The algebra is therefore equivalent to (9.1), provided of course that the field contains the roots of $\varphi$. The converse theorem is obvious.

In conclusion we remark that, if the roots of $\varphi(\xi)$ are not simple, it can be shown in much the same way as in algebras of finite rank that $A$ then possesses a nilpotent invariant subalgebra; also, even if $\varphi(\xi)$ has no roots in the given field, it easily shown that $A$ possesses at least one idempotent element.

* Cf. these Transactions, vol. 16 (1915), p. 329.

Princeton University,

Princeton, N. J. 